ICN-UNAM-01-18

\title{
Quantum Collapse of a Small Dust Shell
}

\author{
A. Corichi, ${ }^{1}$ 用 G. Cruz, ${ }^{2}$ A. Minzoni, ${ }^{2}$ P. Padilla, ${ }^{2}$ M. \\ Rosenbaum, ${ }^{1,7}$ M.P. Ryan, Jr, ${ }^{1}$ N.F. Smyth, ${ }^{3}$ and T. Vukasinac ${ }^{4}$ \\ ${ }^{1}$ Instituto de Ciencias Nucleares - FENOMEC \\ Universidad Nacional Autónoma de México \\ A. Postal 70-543, México D.F. 04510, México. \\ ${ }^{2}$ FENOMEC-IIMAS \\ Universidad Nacional Autónoma de México \\ A. Postal 20-726, México D.F. 04510, México. \\ ${ }^{3}$ Department of Mathematics and Statistics, \\ University of Edinburgh, The King's Building, Mayfield Road, \\ Edinburgh, Scotland, United Kingdom EH9 3JZ. \\ ${ }^{4}$ Departamento de Fisica, Universidad Michoacana de \\ San Nicolas de Hidalgo, Morelia, Michoacan, México.
}

(Dated: October 29, 2018)

\begin{abstract}
The full quantum mechanical collapse of a small relativistic dust shell is studied analytically, asymptotically and numerically starting from the exact finite dimensional classical reduced Hamiltonian recently derived by Hájíček and Kuchař. The formulation of the quantum mechanics encounters two problems. The first is the multivalued nature of the Hamiltonian and the second is the construction of an appropriate self adjoint momentum operator in the space of the shell motion which is confined to a half line. The first problem is solved by identifying and neglecting orbits of small action in order to obtain a single valued Hamiltonian. The second problem is solved by introducing an appropriate lapse function. The resulting quantum mechanics is then studied by means of analytical and numerical techniques. We find that the region of total collapse has very small probability. We also find that the solution concentrates around the classical Schwarzschild radius. The present work obtains from first principles a quantum mechanics for the shell and provides numerical solutions, whose behavior is explained by a detailed WKB analysis for a wide class of collapsing shells.
\end{abstract}

PACS numbers: 04.60.Ds, 04.60.Kz

*Electronic address: corichi@nuclecu.unam.mx

${ }^{\dagger}$ Electronic address: mrosen@nuclecu.unam.mx 


\section{INTRODUCTION}

It is sometimes believed that the study of symmetry-reduced models, or mini-superspace models are of some importance in the understanding of the Physics in the strong curvature limit. This would be true, for instance, in the Big Bang or at the singularity formed by collapse of matter. There are a number of reasons why one would like to study the problem of the quantum collapse of a localized accumulation of matter. For large accumulations one might wish to understand the complete evolution of a collapsing system. A star, for example, might be expected to collapse through its horizon to form a black hole. The usual argument is that this ends all possibility of knowing what happened to the original star. However, the process of Hawking radiation will eventually bleed energy from the black hole, and, if quantum gravity is not taken into account, the original hole will disappear, with the consequent loss of coherence of the original system.

One suggestion that avoids this problem is to assume that any curvature singularities inside the horizon are artefacts of assuming that gravity is a purely classical system, and that quantum gravity could replace these by some quantum state that shows no such singularity. When Hawking radiation has reduced the black hole to the point where quantum gravity comes into play, one might expect to find some sort of quantum remnant that might solve the problem of evaporation. This problem, even if we simplify the system drastically before quantization, is of daunting complexity, involving successively, classical collapse, quantum field theory on a curved background, and finally full quantum gravity coupled to quantum matter.

A simpler problem is to consider the pure quantum collapse of phenomenological matter uncoupled to any field that allows radiation. While in general this involves turning off natural couplings, there is one reduced system that allows us to avoid radiative modes, at least classically. This is the classical collapse of a spherically symmetric accumulation of dust. Birkhoff's theorem shows that there is no gravitational radiation, and the dust matter has no radiative component (even though one might expect the expulsion of shells of matter). An even more drastically reduced case is the collapse of a spherical shell of dust, where the matter is constrained to lie on a (dynamical) spherical shell, thus avoiding blow off problems. This system provides perhaps the simplest quantum collapse scenario, where the effects of quantum gravity are uncontaminated by possible radiative effects. In principle one could use this model to study the collapse of a large shell that would pass through its horizon while still classical and evolve into some sort of quantum object near the classical curvature singularity. Without Hawking radiation this quantum object would always be hidden from outside observers, but it might give us some insight into the avoidance of singularities due to quantum effects.

Note, however, that if one treats the Schwarzschild mass of the shell as a $c$-number (as we will below) [20], the mass will probably have a limit beyond which there is no valid solution to the quantum problem. This phenomenon would be the analogue of the limit of the charge of the nucleus in the relativistic hydrogen atom problem [1]. It is possible to make rough estimates of this limit. Thus, if we consider a particle of mass $m$ orbiting in the gravitational field of a shell of mass $M$, the particle will be quantum mechanical if the radius of the shell is less that the gravitational Bohr radius of the particle, $a_{0}^{G}=\hbar^{2} / G M m^{2}$. It also seems reasonable to demand that $a_{0}^{G}$ be larger than the Schwarzschild radius of the shell for the particle to be observable (note that this excludes the large-mass collapse scenario defined above), i.e. $a_{0}^{G} \geq G M / c^{2}$ (we ignore factors of order one). It is usual to argue that single- 
particle quantum mechanics is no longer valid if $a_{0}^{G}$ is less that the Compton wavelength of the particle, $\hbar / m c$. The second and third of these conditions both give $\sqrt{M m} / M_{P} \leq 1$, where $M_{P}$ is the Planck mass. For stellar mass shells and elementary particles this bound is strongly violated, and elementary particles are produced well inside the horizon. For shell of roughly asteroid size $\left(10^{17} \mathrm{gm}\right)$, the bound is saturated when the shell radius is roughly the Compton wavelength of the electron. We would expect production of light particles well outside the Planck length, and our single-particle quantum mechanics would break down. However, since we exclude all fields, we might hope that our formalism would hold until the mass of the particles produced was equal to that of the shell. In that case, the bound would be saturated when the shell mass was equal to the Planck mass (and the Schwarzschild radius equal to the Planck length). Thus we expect, in order for quantum mechanics to make sense, a limit on the mass of the shell of roughly the Planck mass, similar to a sufficient condition for self adjointness of the quantum operators given by Hájičcek [2] for one of the models we will discuss below.

Keeping in mind these caveats, we will investigate the quantum mechanical collapse of a roughly Planck mass shell of dust, from a rather novel point of view of assymptotics.

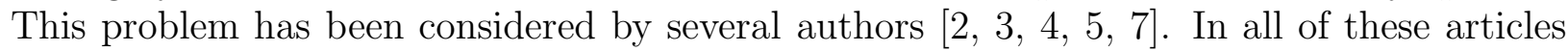
the Israel junction conditions [6] were used to obtain a classical equation of motion for the curvature radius of the collapsing shell relative to the proper time of an observer on the shell. Once the equations were obtained, several possible Hamiltonians leading to these equations of motion were proposed and used to study the corresponding quantum mechanics. These Hamiltonians are both local and non-local. A fairly complete study of a local Hamiltonian is reported in [1]. The main conclusion of the above cited works is the existence of a discrete spectrum of bound states and a set of continuum eigenstates. It was also shown that the ground state hovers away from the classical Schwarzschild radius 四, as might be expected from our qualitative arguments. For the non-local formulation similar results have been obtained, but they are less complete [2, 8]. In this case the spectrum again turns out to be mixed but there is no detailed analysis given of the eigenfunctions. All these quantizations have qualitatively similar spectral properties.

It is also important to remark, however, that all the above work, excepting [3], is not based on the Hamiltonian arising from the spherical reduction of the action of general relativity. Thus the question of how to perform and study the canonical quantization remains open. An important step in this direction is provided by the work of Kuchař and Hájíček [3, []. In this paper the authors obtain the classical equations of motion of the shell as the exact finite dimensional reduction of the full relativistic Hamiltonian.

The purpose of the present work is to continue the program outlined in [3] and investigate the quantum mechanics of the Hamiltonian of Hájiček, Bičák and Kuchař. (A variational procedure for deriving such a Hamiltonian has also been considered recently in [9]). The first difficulty one must overcome arises from the multivalued nature of the canonical Hamiltonian. This leads us to adopt a procedure of quantization which takes into account the relative size of the action on the different sheets of the Hamiltonian. When only the dominant action is taken into account, the problem can be formulated in terms of a non-local Hamiltonian. In this formulation the factor ordering of operators imposed by the requirement of dilational symmetry of the half line (which is the space of values of the shell radius) forces a definite choice of time, which is related to but not the same as the proper time on the shell.

Our procedure results in a non-local Schrödinger equation with vanishing coefficients 
at the classical "Schwarzschild radius" of the shell. Since our quantum mechanics relates to the internal parameters of the shell, with no reference to the external geometry, we will use the term "Schwarzschild radius" to mean the point where the curvature radius of the shell is equal to a constant of motion that is the Schwarzschild mass of the classical exterior geometry. The wave function that solves our non-local Schrödinger equation can be interpreted as the probability density of finding the shell with a given curvature radius. It is interesting to observe that non-local operators often appear in wave problems [10], while the vanishing of the main operator at the "Schwarzschild radius" is analogous to critical layer absorption in fluid mechanics [14], so the equations considered here combine two features which in classical fluid problems are separate. For these reasons, modern ideas of asymptotics 110, 11] can be used successfully to obtain accurate information about the dynamics generated by our new nonlocal Schrödinger equation.

The analysis of the solutions of the above mentioned quantum equation for the shell radius is performed using a generalization to non-local problems of WKB ideas [11]. The use of WKB ideas in a similar context in General Relativity has been explored in [13, 15, 16, 17]. In [15] expected values of interesting physical parameters are calculated using the WKB ground state. While in [13, 16, 17] tunelling amplitudes are computed for simple non-local operators. In the present work we perform a uniform WKB analysis of the continuum and discrete spectrum in order to study the full initial value problem for a collapsing shell. We find that there is indeed a phenomenon analogous to critical layer absorption, which decreases the amplitude of the eigenfunctions and the scattering states beyond the classical Schwarzschild radius. Moreover, we give a complete qualitative description of the dynamics. The analysis is complemented by a detailed numerical study (based on classical ideas in wave propagation [12 adapted to the present situation) for a representative range of parameters. The numerics give solutions that closely match the dynamics that was predicted qualitatively by the asymptotic analysis, and the results show a decomposition of the initial condition into bound states (which concentrate outside the "Schwarzschild radius") and a small probability current leaking to infinity. We also show how the present model explains, due to the presence of a critical-layer like behavior, the concentration of the solution of an initial value problem, with initial values outside the "Schwarzschild radius", in the region outside this radius.

To complete our analysis, we compare our numerical solutions with those obtained for a simpler non-local ad hoc model [2, 5] and contrast their differences. Finally we give arguments for how the present formulation could be used to obtain local information (around the space-time trajectory of the shell) about the space-time geometry.

The paper is organized as follows: In the second section we review the variational formulation of the classical relativistic problem. The third section studies the classical motion and solves the problem of canonically quantizing the multivalued Hamiltonian. This section ends with a canonical quantum formulation in terms of a non-local equation for the shell problem. In the fourth section the Hamiltonian operator is analyzed both asymptotically and numerically. The fifth section is devoted to the detailed numerical study of the dynamics for representative values of the parameters and initial conditions. The last section is dedicated to conclusions and suggestions for further work. The non-standard details of matching for the WKB solution are sketched in the Appendix. 


\section{FORMULATION OF THE PROBLEM}

In order to make our presentation as self contained as possible, as well as to fix notation and conventions, we recall briefly some of the details of the Kuchař-Hájíček [3, 7] construction of the canonical dynamics of gravitational shells. The geometry of the shell is described by

$$
d s^{2}=-\Lambda^{2} d t^{2}+R^{2}(t) d \Omega^{2}
$$

where the function $R(t)$ is the (area) radius of the shell and $\frac{d \tau}{d t}=\Lambda$ with $\tau$ denoting the proper time on the shell.

The Lagrangian for the dust matter is taken to be of the form

$$
\mathcal{L}_{\Sigma}^{M}=\int \frac{1}{2} M\left(\Lambda^{-1} \dot{T}^{2}-\Lambda\right) d t
$$

where $M=4 \pi R^{2} \rho(t)$ is the total mass of the shell and $\dot{T}=-U_{t}$ is the temporal component of the dust velocity [3].

The gravitational field Lagrangian is expressed in terms of the variables $V=\frac{\dot{R}}{\Lambda}$ - the proper velocity - and $F_{ \pm}=1-\frac{2 M_{ \pm}}{R}$, where the Schwarzschild mass $M_{ \pm}$can be different on each side of the shell. For the special case of a collapsing shell $M_{-}=0$, so the space inside the shell is flat. Thus

$$
\mathcal{L}_{\Sigma}^{G}=\int\left(M_{+} \dot{T}_{+}+[L]\right) d t
$$

Here $\dot{T}_{+}$is the Schwarzschild time which determines the embedding of the shell in the space to the right of the shell. The function $L$ is given by

$$
L=\Lambda R\left[\left(F+V^{2}\right)^{\frac{1}{2}}-V A\left(|F|^{-\frac{1}{2}} V\right)\right]
$$

where

$$
\begin{aligned}
A(\eta) & =\sinh ^{-1} \eta, \text { for } F>0 \\
& =\operatorname{sgn}(\eta)\left|\cosh ^{-1}\right| \eta|| \text { for } \quad F<0 .
\end{aligned}
$$

The jump $[L]$ is obtained by using $F_{+}=1-\frac{2 M}{R}$ on the right of the shell and $F_{-}=1$ on the left.

The total action is given by

$$
\mathcal{L}=\mathcal{L}_{\Sigma}^{G}+\mathcal{L}_{\Sigma}^{M}
$$

It is now possible to obtain the equations of motion. Variation with respect to $M$ gives $\Lambda=\dot{T}$. Variation with respect to $T$ gives $\dot{M}=0$. Variation with respect to $T_{+}$gives $M_{+}=$ constant. Finally, variation with respect to $M_{+}$gives $\dot{T}_{+}=\Lambda F^{-1} \sqrt{F^{2}+V^{2}}$. The equation of motion is obtained by varying with respect to $\dot{R}$. The energy condition which determines the trajectories for a given proper mass $\mathcal{M}$ of the shell is

$$
\sqrt{1-\frac{2 M}{R}+V^{2}}-\sqrt{1+V^{2}}=-\frac{\mathcal{M}}{R} .
$$

The relative signs of the square roots are obtained from the requirement that the shell motion generate a cup space-time [3]. 
This equation is taken as the starting point in the treatments in Refs. [4, 5]. In these works there are several possible Hamiltonians that produce the equations of motion (8). However, The resulting quantizations, as we have pointed out in the Introduction, are not obtained from an exact dimensional reduction of the full general relativistic Hamiltonian of the theory. This leaves open the question (since the quantizations are not unitarily equivalent) of which canonical quantization one should use, and how the properties of these quantizations differ from those of ad hoc procedures. In the first place, one must obtain the classical Hamiltonian from the Lagrangian (4). This is done in [3] by finding the appropriate canonical momentum and then constructing, by a finite dimensional reduction, the Hamiltonian.

The Legendre transformation is given in [3]. Note that what was done there is equivalent to the full ADM procedure. The gravitational action leads to a Hamiltonian constraint instead of a Hamiltonian. By choosing an internal time, we can solve the constraint for the momentum conjugate to our time, which gives us a straightforward Hamiltonian (the ADM Hamiltonian). Classically, of course, this procedure is exactly equivalent to solving the unconstrained system and applying the constraint. Quantum mechanically there is an enormous difference, one procedure leading to an ADM Hamiltonian and the other to a Wheeler-DeWitt equation. In the ADM procedure, there is no guarantee that the evolutions for different times and different Hamiltonians are unitarily equivalent. The Wheeler-DeWitt route leads to the well-known "problem of time" in the solutions and difficulties in defining probabilities. Since we will use the ADM procedure and two different times in this article, we will briefly discuss problems associated with our approach. For the moment we will just give the results from [3] necessary for our purposes.

First, the expression for the canonical momentum $\tilde{P}=\frac{\partial L}{\partial V}$ must be inverted to obtain $V$ as a function of $\tilde{P}$. It turns out that for $0 \leq R \leq 2 M$ the inverse function is double valued (see Equations (C33)and(C34) in [3]). This poses a question of interpretation when using $\tilde{P}$ instead of $V$. In Ref. [3] the total Hamiltonian constraint for the shell plus gravity takes the form

$$
H^{G}+\mathcal{M}=0
$$

where the gravitational super-Hamiltonian $H^{G}$ is written in terms of $\tilde{P}$ and $R$. Since $\mathcal{M}$ is the conjugate variable to $\tau$, the proper (or curvature) time on the shell, the natural time choice is $\tau$, which gives a Hamiltonian $\mathcal{M}=H^{G}$. In Ref. [3] there are several possible values for $H^{G}$ depending on whether $R$ is greater than or less than $2 M$. We must construct the appropriate single valued function from these for a viable classical mechanics. To do this it is only necessary to construct the two-sheeted surface joined across the branch cut $0 \leq R \leq 2 M$ at $\tilde{P}=0$.

The patching condition across the cut (analytic continuation) is found by taking into account the fact that $V$ does not change sign across the cut Thus we have for $R \geq 2 M$ that the Hamiltonian is given by

$$
H_{1}^{G}=-\sqrt{2} R\left(1-\frac{M}{R}-\sqrt{1-\frac{2 M}{R}} \cosh \frac{\tilde{P}}{R}\right)^{1 / 2} .
$$

For $0 \leq R \leq 2 M$ and $\tilde{P}>0$ the Hamiltonian is given by

$$
H_{2}^{(+) G}=-\sqrt{2} R\left(1-\frac{M}{R}-\sqrt{\left|1-\frac{2 M}{R}\right|}\left|\sinh \frac{\tilde{P}}{R}\right|\right)^{1 / 2}
$$




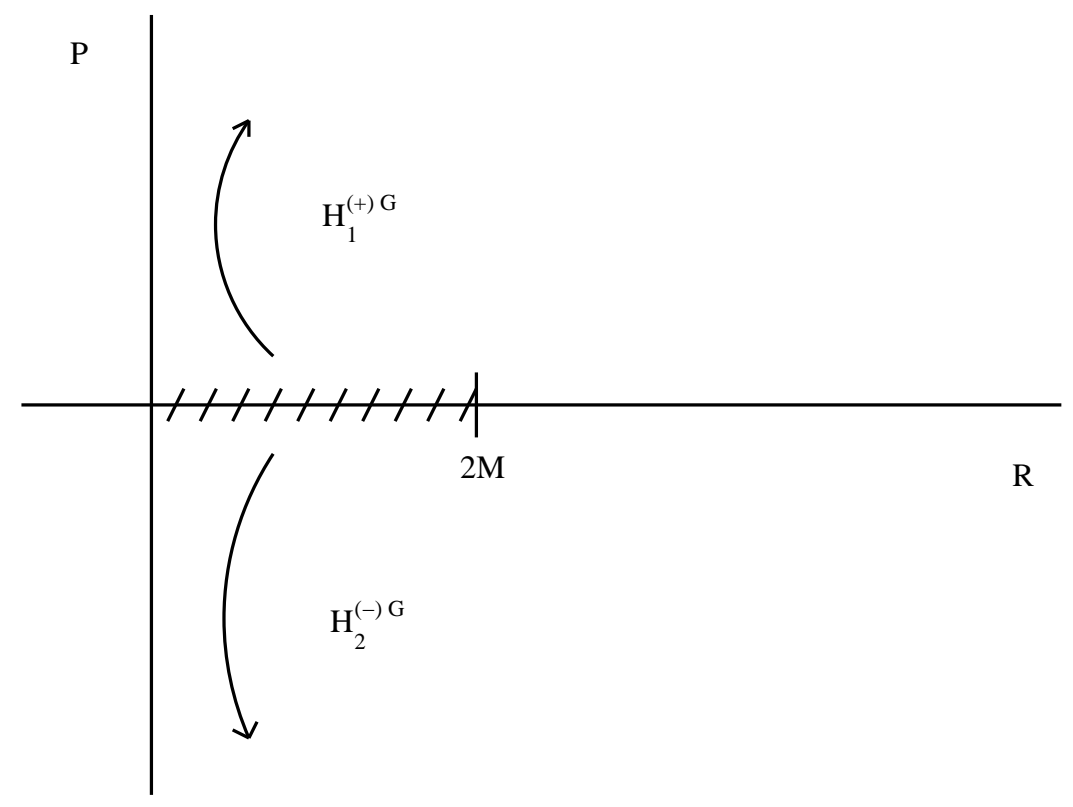

FIG. 1: $R-P$ plane with cut in $0 \leq R \leq 2 M$.

where the argument is positive. This is joined smoothly at $P=0$ with

$$
H_{2}^{(-) G}=-\sqrt{2} R\left(1-\frac{M}{R}+\sqrt{\left|1-\frac{2 M}{R}\right|}\left|\sinh \frac{\tilde{P}}{R}\right|\right)^{1 / 2},
$$

where the corresponding argument is positive and $\tilde{P}<0$. Likewise, the other connection goes from $\tilde{P}<0$ to $\tilde{P}>0$ through the other sheet. The points on the different sheets are then projected onto the $R-\tilde{P}$ plane to obtain the classical orbit. This is illustrated in Fig. 1 .

It is to be noted that the double valuedness does not stem from the square root but from the $\tilde{P} \rightarrow V$ transformation. Also note that in the Legendre transformation the signs of $V$ and $\tilde{P}$ are not necessarily the same. The velocity $V$ does not necessarily change sign on a given orbit when $\tilde{P}$ does (although in some cases it does), due to the transformation. Thus the Legendre transformation is only possible in terms of the double valued Hamiltonian just described. As we shall see in the following Section, this will have non-standard implications for the quantum theory based on this Hamiltonian.

Notice that it is not necessary to interpret the geometrical variable $\tau$ as time. The constraint (9) is simply a linear combination of the geometrical variables $H^{G}$ and $\mathcal{M}$.

\section{CLASSICAL ORBITS, ACTION AND QUANTIZATION}

In order to quantize we must first study the classical mechanics of the problem and provide a suitable formulation for the multiple valued Hamiltonian. For the classical mechanics we have to solve for the level lines $H=E$. We anticipate these level lines to lie on two sheets. More specifically, we take $R \geq 2 M$. Thus $H^{G}=E$ gives for the level lines the equation

$$
\cosh \frac{\tilde{P}}{R}=f(E, R)
$$




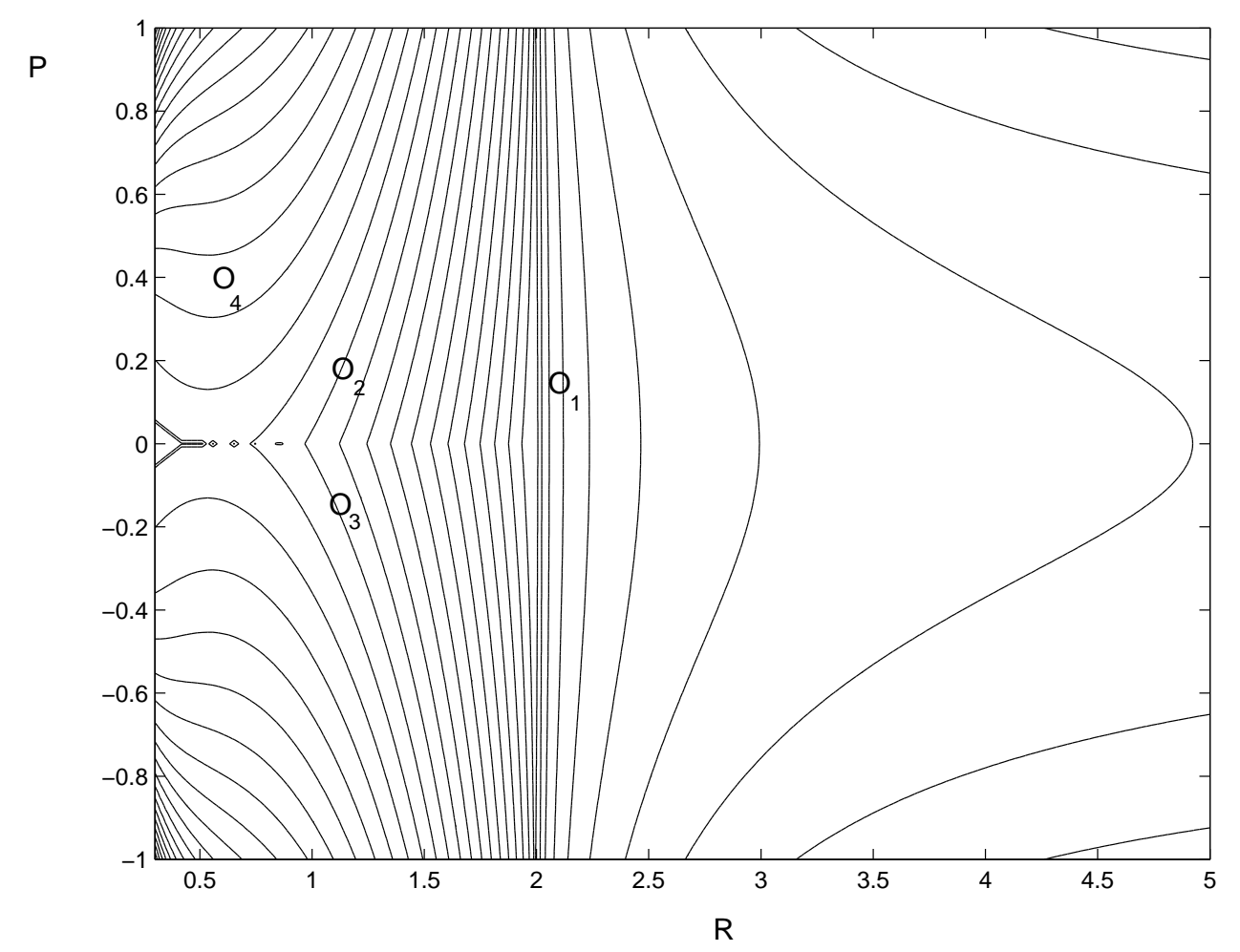

FIG. 2: $(R, P)$ phase plane given by (13), (14), (16) and (18).

where

$$
f(E, R)=\frac{\left(-E^{2} / 2+R^{2}-M R\right)}{R^{3 / 2} \sqrt{R-2 M}} .
$$

Provided $f(E, R) \geq 1$, Eq. (14) can be solved for $\tilde{P}$ real. There are two solutions, one with $\tilde{P}>0$ and the other with $P<0$. As $R \rightarrow \infty$ there is a real solution only for $E^{2} \leq M^{2}$. For $M^{2} \leq E^{2} \leq 2 M^{2}$ the orbits do not extend to infinity; they have a turning point at $R^{*}(E)$ given by $f\left(E, R^{*}\right)=1$, with

$$
R^{*}(E)=\frac{M E^{2}+\sqrt{M^{2} E^{4}+E^{4}\left(E^{2}-M^{2}\right)}}{2\left(E^{2}-M^{2}\right)}=\frac{E^{2}(M+E)}{2\left(E^{2}-M^{2}\right)}>0
$$

for $E^{2} \geq M^{2}$. On the other hand, for $E^{2} \geq 2 M^{2}$ there are no orbits which represent classical trajectories. For these orbits, $\tilde{P} \rightarrow \infty$ as $R \rightarrow 2 M$. This part of the orbit is labeled $O_{1}$ in Fig. 2.

Let us now consider the situation when $R \leq 2 M$. When $R$ approaches $2 M$ from below, we have that the orbits are solutions of

$$
\left|\sinh \frac{\tilde{P}}{R}\right|=f(E, R) .
$$

The solutions match as $|\tilde{P}| \rightarrow \infty$ since in that limit $\left|\sinh \frac{\tilde{P}}{R}\right| \sim \cosh \frac{\tilde{P}}{R}$. Thus the orbits can be continued, and if we pick the collapsing orbits, i.e. $\tilde{P}<0$ they are continued as solutions 
of (16) up to $\tilde{P}=0$. We find that $\tilde{P}=0$ at

$$
R=\tilde{R}(E)=\frac{M+\sqrt{M^{2}+2 E^{2}}}{2},
$$

obtained by solving $f[E, \tilde{R}(E)]=0$. This orbit is labeled $O_{2}$ in Fig. 2. Now, at this point the solution passes to the other sheet and continues as a solution of

$$
\left|\sinh \frac{\tilde{P}}{R}\right|=-f(E, R)
$$

with $\tilde{P}>0$. This is labeled as $O_{3}$ in Fig. 2. The mirror image of the above description applies to the situation for expanding orbits. Finally for the case $E^{2} \leq M^{2}$ the situation is the same as before but now the orbits extend as incoming or outgoing orbits as $R \rightarrow \infty$. These orbits are labeled in Fig. 2 as $O_{4}$. This description reproduces the classical collapse as well as expanding motion with turning points. This is to be contrasted with the phase plane obtained in [17] where the local coordinate system used gives only the $H_{1}^{G}$ portion of the Hamiltonian. There the unbounded orbits represent collapse up to a point and the bounded ones represent expansion and ultimate collapse. There is a separatrix dividing the phase of these two types of motion. Also, in the quantum mechanics developed in the above cited paper there is an exponentially small probability of total collapse. In our analysis, in contrast, the orbits do not have a separatrix and they all show ultimate collapse. We will show later that the collapse turns out to be localized around $R=2 M$ via a phenomenon of critical layer, the nature of which is unraveled by the Hamiltonian that we use. This phenomenon is not captured by the local expansion used in [13, 17]. With this picture we turn now to the problem of quantization.

We will choose our internal time to be the proper time on the shell, $\tau$, and $E$ is its conjugate variable. We must now take $H^{G}$ to be an operator on an appropriate Hilbert space, and $R$ to be a multiplication operator while $\cosh \tilde{P} / R$ and its functions are to be realized as a pseudo differential operator on the Hilbert space [2]. However, in order to carry out this canonical quantization we require a single-valued Hamiltonian, while, as already noted, the true Hamiltonian of the shell given in Equations (11) and (12) is multi-valued. This situation is new on both counts, and because of this we need to introduce a proposal, which we believe to be new, to quantize the system. First we will consider possible definitions of the operator $\cosh \tilde{P} / R$. Hájíček [2] proposes quantizing the shell radius, which is restricted to the half line $R \geq 0$, by considering the Hilbert space of functions $\Psi$ in $L^{2}[0, \infty)$ with respect to the measure $R^{-1} d R$. The observables are taken to be $R$ and $\Pi=-i R \frac{d}{d R}$. The factor ordering is taken to make the operator $\cosh \tilde{P} / R$ formally self-adjoint with $\tilde{P}=\Pi / R$ with respect to the measure $R^{-1} d R$. Since the hyperbolic cosine contains only even powers of its argument, it is sufficient to take a factor ordering that makes $(\Pi / R)^{2}$ formally self adjoint [2]. In our case, due to the $1 / R$ factor, it is easy to check that this ordering is not possible. This forces us, in order to preserve symmetries due to the dilational symmetry of the half line, to redefine the $\cosh \tilde{P} / R$ operator as simply $\cosh P$. The operator $|\sinh P|=\sqrt{\cosh ^{2} P-1}$ can be shown to be self adjoint by means of spectral methods.

This quantization leads us to a new choice of time (lapse $\Lambda$ ) in order to re-scale the momentum. The freedom we have to choose $\Lambda$ allows us to construct a rescaled Hamiltonian and a canonical quantization scheme. Thus if we now take $\Lambda=\mu / R$ in Eq. (11), the Lagrangian (4) becomes

$$
L=\mu\left\{\left(F+V^{2}\right)^{1 / 2}-V F\left(|F|^{-1 / 2} V\right)\right\}
$$


Note that the resulting new canonical momentum obtained from

$$
P=\frac{\partial L}{\partial V}
$$

does not contain the factor $1 / R$. Finally, Eq. (8) is exactly the same, except that now $V=\frac{\dot{R} R}{\mu}$, which allows us to recover $\dot{R}$ from $V$. The Hamiltonian with this new choice of lapse is essentially the same as in Eqs. (10)-(12), except that $\tilde{P} / R$ is replaced by $P$. With our new time, we can obtain (as in Ref. [2]) $\cosh P$ as a formally self-adjoint operator.

The second difficulty we must address is the question of how to handle the multivaluedness of the Hamiltonian. By analogy to the path integral formulation [13], we consider the classical action

$$
\int_{0}^{R} P d R
$$

along an orbit, say $\mathrm{O}_{3}, \mathrm{O}_{2}$, or $\mathrm{O}_{1}$. Clearly the action along the dashed, $\mathrm{O}_{3}$, portion of the orbit is small compared to the action along the $O_{2}$ and $O_{1}$ portions in solid line. This occurs because $R^{*}(E)>>\tilde{R}(E)$. An approach similar in spirit was used in [13] where the action was approximated in the complex plane and then the path integral was evaluated by a saddle point approximation. Here we approximate the real action and obtain an approximate operator equation. Also, since the classical Hamiltonian involves a square root, it is necessary to define first the square and then take the square root using spectral calculus. To this end we begin by defining $H$ as the square of $H^{G}$ in the form

$$
\begin{aligned}
& H=2\left\{\left(R^{2}-M R\right)-R^{3 / 2}|R-2 M|^{1 / 2} \cosh P\right\} \quad R \geq 2 M, \\
& H=2\left\{\left(R^{2}-M R\right)-R^{3 / 2}|R-2 M|^{1 / 2}|\sinh P|\right\} \quad R \leq 2 M,
\end{aligned}
$$

once the small contribution of the $\mathrm{O}_{3}$ portion of the orbit is neglected This form of $H$ introduces classical turning points at $\tilde{R}(E)$ which are not present in the original Hamiltonian. The implications of this fact will be discussed below.

We now turn to the quantization of (21-22). As we have already remarked, the operator $\cosh P$ is transformed into a self-adjoint operator on functions $\Psi$ relative to the measure $d R / R$. A unitary transformation $R^{1 / 2} \Psi \rightarrow \Psi$ turns cosh $P$ into a self-adjoint operator in $L^{2}(0, \infty)$ relative to the measure $d x$. The functions $\Psi$ must have all their even derivatives equal to zero at $x=0$. We thus have

$$
\begin{aligned}
H \Psi(x)=2 & \left\{\left(x^{2}-M x\right) \Psi\right. \\
& \left.-x^{3 / 2}|x-2 M|^{1 / 2} \frac{1}{2 \pi} \int_{-\infty}^{\infty} \cosh k e^{i k x} \hat{\Psi}(k, t) d k\right\}, \\
& x \geq 2 M, \\
H \Psi(x)=2 & \left\{\left(x^{2}-M x\right) \Psi\right. \\
- & \left.x^{3 / 2}|x-2 M|^{1 / 2} \frac{1}{2 \pi} \int_{-\infty}^{\infty}|\sinh k| e^{i k x} \hat{\Psi}(k, t) d k\right\}, \\
& x \leq 2 M,
\end{aligned}
$$

where the Fourier transform $\hat{\Psi}$ of $\Psi$ is defined by

$$
\hat{\Psi}(k, \tau)=\int_{-\infty}^{\infty} e^{-i k x} \Psi(x, \tau) d x .
$$


Now, since $H$ will be shown to be positive, the spectral calculus gives the final equation in the form

$$
i \frac{\partial u}{\partial \tau}=H^{1 / 2} u,
$$

and $u(x, 0)=\Psi(x)$ is the given initial condition. This procedure gives us a well-defined equation for the quantum evolution of the radius of the shell. If one prefers the WheelerDeWitt approach, one need only reinterpret (26) as the equation that result from applying the Hamiltonian constraint (9) as a quantum equation, and think of $\tau$ as simply a gravitational variable. The solutions will be the same.

Before we solve the equation we need to make several remarks. In the first place, note that pseudo-differential equations like (26) arise in classical wave propagation [10], where the nonlocality of the main operator reflects the incompressibility of the fluid. The zero coefficient at $x=2 M$ in front of the main operator also arises in the study of wave propagation in stratified fluids in the presence of inviscid critical layers [14]. However, in the case of critical layers the main operator is local. For these reasons we will use the same analytical and numerical methods developed for those problems, with appropriate modifications to take into account the presence of the vanishing coefficient multiplying the main nonlocal operator in order to solve the problem posed in (26).

One final remark is that we will treat $M$ as a $c$-number similar to the charge on the electron in the hydrogen atom problem. Actually there is no reason for this since $M$ is merely a constant of motion, that is, a phase space function or $q$-number. A simple analogue might be a particle of mass $m$ moving in two dimensions, where in one of the directions, $z$, the particle is free, and in the other, $y$, moves in a harmonic oscillator potential. The eigenfunctions have the form $e^{i p_{z} z} \psi_{n}\left(y, p_{z}\right)$, where the $\psi_{n}$ are harmonic oscillator eigenfunctions. We may consider an eigenstate of $p_{z}$ and study the evolution of $\psi(y, t)$ with $p_{z}$ constant, but there is no reason to do so. One can just as well study more general functions of the form $\int f\left(p_{z}\right) e^{i p_{z} z} e^{-i \frac{p_{z}^{2}}{2 m} t} \psi\left(y, p_{z}\right) e^{-i E_{n} t} d p_{z}$. In our case, we have taken an eigenfunction of $M, \delta(M-$ $\left.M_{0}\right)$, but one should study more general situation.

Finally, we compare the solution of Equation (26) with another model studied in [2, 8],

$$
i \frac{\partial u}{\partial t}=\frac{1}{2 \pi} \int_{-\infty}^{\infty} \cosh k e^{i k x} \hat{u}(k, t) d k-\frac{\beta u}{x} .
$$

Note that further other models considered in the literature [5] have similar structures with different symbols for the nonlocal operator, or are similar to (27) but with functions of local operators. The cosh $k$ terms appear in all models, since a proper time variable is used in their description. The coordinate system chosen to cross the classical horizon produces the nonlocal terms. The potential term is common to all the models. However, the exact reduction leading to (26) has the new feature of having a vanishing term multiplying the main operator. We will discuss the implications of the similarities and differences between the models in the following sections.

\section{WKB SOLUTIONS, SPECTRA AND THE QUALITATIVE BEHAVIOR OF THE SOLUTIONS}

As $x \rightarrow 2 M$ the term in front of the nonlocal operator in (23) - 24 produces high frequency spatial oscillations. Because of the non-locality we look for an approximate solution 
in the WKB form of (26). We take

$$
u=A(x) e^{i \theta(x)} e^{-i E \tau}
$$

The phase $\theta$ satisfies the eikonal equation (13) where $\tilde{P} / R$ is replaced by $\theta^{\prime}$ (since $\tilde{P} / R$ is replaced by $P$ in the new formulation), and the derivatives of $A$ are assumed to be small relative to $\theta^{\prime}$. The eikonal equation and amplitude equation are readily obtained from the average Lagrangian [10, 11]

$$
\iint\left[i\left(u^{*} \partial_{\tau} u-u \partial_{\tau} u^{*}\right)+u^{*} H u\right] d x d t
$$

The eikonal equation takes the form

$$
\begin{gathered}
\cosh \theta^{\prime}=\frac{-E^{2} / 2+x^{2}-M x}{x^{3 / 2}|x-2 M|^{1 / 2}}=f(E, x), \quad x \geq 2 M \\
\left|\sinh \theta^{\prime}\right|=\frac{-E^{2} / 2+x^{2}-M x}{x^{3 / 2}|x-2 M|^{1 / 2}} . \quad x \leq 2 M
\end{gathered}
$$

The first equation can be solved for real $\theta^{\prime}$ (which represents an oscillatory solution as $x \rightarrow \infty)$ provided $f(E, x) \geq 1$. This occurs for $x>0$ provided $E^{2} \leq M^{2}$. On the other hand, when $E^{2} \geq M^{2}$ real solutions are only possible if $2 M \leq x \leq R^{*}(E)=\frac{E^{2}(M+E)}{2\left(E^{2}-M^{2}\right)}$. Beyond $R^{*}$ the solution becomes complex, and this region, $M^{2} \leq E^{2} \leq 2 M^{2}$, provides candidates for the point spectrum. On the other hand, the region $0 \leq E^{2} \leq M^{2}$ provides candidates for scattering states. Note that $E \leq 0$ is not in the spectrum. In the region $\tilde{R}(E) \leq x \leq 2 M$ where $\tilde{R}(E)=\frac{1}{2}\left(M+\sqrt{M^{2}+2 E^{2}}\right)$, Eq. (31) has real solutions. Even though $\theta^{\prime}$ goes to infinity as $x \rightarrow 2 M$ from above or below, $\theta^{\prime}$ matches, since $\cosh \theta^{\prime} \sim\left|\sinh \theta^{\prime}\right|$ as $\left|\theta^{\prime}\right| \rightarrow \infty$. For $0<x<\tilde{R}(E), \theta^{\prime}$ is again complex. This produces an exponentially decaying solution as $x \rightarrow 0$. This is a consequence of the approximation we have used, which replaces a small action by zero in the region $0 \leq x \leq \tilde{R}(E)$. Also note that when $E^{2}<0$ there are no solutions defined in the whole interval. This shows that $H$ is indeed positive.

Given the phases, the amplitudes are determined from the average Lagrangian by varying with respect to $\theta^{\prime}$. Using

$$
\int\left[H\left(\theta^{\prime}\right) A A^{*}-E A A^{*}\right] d x
$$

we find that

$$
\frac{\partial}{\partial x}\left(|A|^{2} H\left(\theta^{\prime}\right)\right)=0
$$

which gives $|A|^{2}=$ constant $/ H\left(\theta^{\prime}\right)$, where in each region $\theta^{\prime}$ is chosen according to Eqs. (30) or (31).

We now give the explicit expressions for the scattering states and for the bound states. We begin by finding the phases. For $\tilde{R}(E) \leq x \leq 2 M$ we have

$$
\begin{aligned}
\theta_{1}(x)= & \int_{\tilde{R}(E)}^{x} \ln \left\{\sqrt{\frac{\left(\xi^{2}-M \xi-E^{2} / 2\right)^{2}}{\xi^{3}(\xi-2 M)}+1}\right. \\
& \left.+\frac{\left(\xi^{2}-M \xi-E^{2} / 2\right)}{\xi^{3 / 2}|\xi-2 M|^{1 / 2}}\right\} d \xi,
\end{aligned}
$$


while for $2 M \leq x$ we have

$$
\begin{aligned}
\theta_{2}= & \theta_{1}(2 M)+\int_{2 M}^{x} \ln \left\{\sqrt{\frac{\left(\xi^{2}-M \xi-E^{2} / 2\right)^{2}}{\xi^{3}(\xi-2 M)}-1}\right. \\
& \left.+\frac{\left(\xi^{2}-M \xi-E^{2} / 2\right)}{\xi^{3 / 2}(\xi-2 M)^{1 / 2}}\right\} d \xi .
\end{aligned}
$$

Note that when $E^{2} \leq M^{2}, \theta_{2}(x)$ is defined for all $x>2 M$. On the other hand, when $E^{2} \geq M^{2}, \theta_{2}(x)$ is real only up to $x=R^{*}(E)$. The amplitude associated with $\theta_{1}$ is given by

$$
A_{1}=\frac{1}{\left[\left(-E^{2} / 2+x^{2}-M x\right)^{2}+x^{3}|x-2 M|\right]^{1 / 4}}, \quad \tilde{R} \leq x \leq 2 M
$$

while that associated with $\theta_{2}$ is

$$
A_{2}=\frac{1}{\left[\left(-E^{2} / 2+x^{2}-M x\right)^{2}-x^{3}|x-2 M|\right]^{1 / 4}} . \quad 2 M \leq x
$$

Note that when $x=2 M, A_{1}=A_{2}$. Also, when $0 \leq E^{2} \leq M^{2}, A_{2}$ is always well defined for $x \geq 2 M$ and as $x \rightarrow \infty$ it is given by $A_{2}(x) \sim \frac{1}{x^{1 / 2}}$. On the other hand, when $E^{2} \geq M^{2}$, as $x \rightarrow R^{*}(E), A_{2} \rightarrow \infty$. This indicates, as usual [10, 11], the presence of a transition region which has to be included in order to construct the exponentially decaying eigenfunctions of the point spectrum. Finally, as $x \rightarrow \tilde{R}(E)$, the amplitude $A_{1}$ is finite. Due to the nonanaliticity of $\left|\sinh \theta^{\prime}\right|$ at $\theta^{\prime}=0$ when $x=\tilde{R}(E)$, the behavior for $x<\tilde{R}(E)$ will be shown in the Appendix to be algebraically small. With these considerations we can now construct the WKB functions which represent both scattering states and bound states.

We begin with the scattering states. From the Appendix we have that the solution for $x \sim \tilde{R}(E)$, but less that $\tilde{R}(E)$, matches to the solution

$$
A_{1}(x) \sin \theta_{1}(x)
$$

In turn, this continues into

$$
A_{2}(x) \sin \theta_{2}(x),
$$

where $A_{1}, \theta_{1}, A_{2}, \theta_{2}$ are given by Eqs. (34)-(37). Thus the function $\varphi(x, E)$ defined by Eqs. (38) and (39) is a solution of the eigenvalue equation that is not square integrable and, as expected, represents a perfectly reflected probability wave impinging from infinity.

The bound states occur for $E^{2} \geq M^{2}$. In this case, as already noted above, $A_{2} \rightarrow \infty$ as $x \rightarrow R^{*}(E)$. This is typical turning point behavior, and the appropriate transition function will be shown in the Appendix to be the Airy function. Beyond $R^{*}(E)$ the phase is complex and the solution decays exponentially. The matching condition between the oscillating and the Airy function gives the usual Bohr-Sommerfeld condition for the eigenvalue $E$. It takes the form

$$
\theta_{2}\left[R^{*}(E)\right]=\left(n+\frac{1}{2}\right) \pi
$$

This provides a transcendental equation for $E$. It is clear from (40), since $\theta_{2}\left[R^{*}(E)\right] \rightarrow \infty$ as $E^{2} \rightarrow M^{2}$ from above, that there are infinitely many eigenvalues accumulating at $M^{2}$ from above. An illustration of the W.K,B. eigenfunctions is given in Fig. (3). Notice that the oscillations are concentrated at the "critical layer," $x \sim 2 M$. It is also important to notice 
that the size of the support of the oscillatory part of the eigenfunction which extends up to $R^{*}(E)$ increases very rapidly even for low eigenvalues $(n=3,4)$.

The eigenvalues of $H$ are the solutions to (40) and the continuous spectrum extends from zero to $M$. The analysis just presented shows also explicitly that the eigenfunctions are localized around $R=2 M$ because of the critical layer behavior. Again, the existence of a point spectrum here is to be contrasted with the results of [17 where the spectrum is only continuous. This effect was also found in other model Hamiltonians [4], but no intrinsic explanation was possible. Finally, our spectrum and eigenfunctions must be contrasted with those of other models. In all models, including the present one, the spectrum is always mixed. However, previous models have an unbounded continuous spectrum, and their point spectrum has a ground state [2, 4, 8]. This situation just reflects the fact that previous models were based on a choice of an ad hoc Hamiltonian that reproduced the equation of motion. Note, nonetheless, that in principle the inverses of these ad hoc Hamiltonians are equally legitimate choices, as they also reproduce the classical equations of motion, but their spectrum will have a bounded continuous part that is below the point spectrum. Thus even if the quantum problems are not unitarily equivalent, they have the same spectral properties as the ones obtained from the finite dimensional reduced relativistic Hamiltonian. On the other hand, the form of the eigenfunctions in those models was found to be similar to the ones for the hydrogen atom [4, 8], while for the Hamiltonian considered here the eigenfunctions and the scattering states have a large number of oscillations concentrated around $x=2 M$.

In the following section we consider the quantum dynamics generated by the Schrödinger equation (26) and compare it with the dynamics of the non-local Hamiltonian considered in [2, 4, 5] and which leads to

$$
i \frac{\partial u}{\partial t}=\frac{1}{2 \pi} \int_{-\infty}^{\infty} \cosh k e^{i k x} \hat{u}(k, t) d k-\frac{\beta u}{x},
$$

However, before closing this section we would like to make some remarks concerning the spectrum problem associated with (41) which show that its eigenvalues and eigenfunctions behave as hydrogen-like. Thus taking $u=v \exp (-i E t)$, the eigenvalue equation becomes

$$
\hat{E} v=\frac{1}{2 \pi} \int_{-\infty}^{\infty} \cosh k e^{i k x} \hat{v}(k, t) d k-\frac{\beta v}{x} .
$$

The WKB eigenfunctions take the form

$$
v=A e^{i \theta(x)}
$$

and the phase $\theta$ satisfies

$$
\cosh \theta^{\prime}=E+\frac{\beta}{x}
$$

Consequently for $E \geq 1$ there are always two real solutions for Eq. (44), and the case $E \geq 1$ is always part of the continuous spectrum with eigenfunctions perfectly reflected at $x=0$. Alternatively, when $E<1$ there are turning points for

$$
E+\frac{\beta}{x}=1
$$

which gives $x(E)=\frac{\beta}{(1-E)}$. The quantization rule for the point spectrum is

$$
\int_{0}^{x(E)} \cosh ^{-1}\left(E+\frac{\beta}{x}\right) d x=\left(n+\frac{1}{2}\right) \pi .
$$


This equation shows that since $x(E) \rightarrow \infty$ as $E \rightarrow 1$, there are infinitely many eigenvalues accumulating from below at $E=1$. Also for $E=0$ the integral is smaller than $\frac{\pi}{2}$. This implies that the spectrum is bounded from below. We thus see that the eigenvalues and eigenfunctions of (42) behave indeed as hydrogen-like (see also [8]). With this information we shall next consider numerical solutions of (41) and (26) and provide an interpretation of the results.

\section{QUANTUM DYNAMICS AND NUMERICAL SOLUTIONS}

This section has three parts. In the first one, we focus on the general structure of the solutions of the quantum problem and show that, in certain regime of interest, the quantum dynamics can be approximated by a simpler Hamiltonian. In the second part, we describe the numerical scheme used in the calculation. In the last part, we describe the numerical solutions for different initial conditions and compare them with the WKB solutions previously studied.

\section{A. Spectral properties and analytical results for the dynamics}

Let us begin by first remarking on the basic structure of the solutions of the Schrödinger equations for the non-local Hamiltonians (23) and (24). The solutions of the initial value problem take the form

$$
u(x, \tau)=\sum_{n=1}^{\infty} c_{n} \psi_{n}\left(x, E_{n}\right) e^{-i E_{n} \tau}+\int_{0}^{M} c(E) \psi(x, E) e^{-i E \tau} d E .
$$

The $c_{n}$ and $c(E)$ are just the projections of the initial condition on the discrete and continuous eigenfunctions respectively. The second term in (47) decays in time as $\tau^{-1 / 2}$. It is important to notice from equation (34) that all the states in the continuous spectrum have high wavenumbers in the vicinity of the critical layer. It is therefore expected that the radiation produced will be of short wavelength in the vicinity of $2 M$ and of long wavelength as $x \rightarrow \infty$. Since the eigenfunctions are strongly localized between $R^{*}\left(E_{n}\right)$ and $\tilde{R}\left(E_{n}\right)$, the first term is localized. Since $\psi \approx 0$ as $x \rightarrow 0$ the dynamics will show small probability of total collapse.

We have shown that the discrete spectrum is concentrated around $E \sim M$, while the continuous spectrum only contributes to the decaying part of the solution. Thus the solution is dominated (in particular for large time which is the relevant behavior for the physics of collapse) for values of the spectral variable close to $M$. We can take advantage of this fact in order to simplify considerably the numerical integration of (26). To achieve this

simplification use $\hat{H}^{1 / 2}=\sqrt{\left(\hat{H}^{1 / 2}\right)^{2}}$. Using the spectral representation in terms of the eigenfunctions, we have

$$
\hat{H}^{1 / 2} \varphi(x)=\sum_{n} \sqrt{E_{n}^{2}} c_{n} \psi_{n}\left(E_{n}, x\right)+\int_{0}^{M} \sqrt{E^{2}} c(E) \psi(E, x) d E .
$$

Now, since the spectrum is concentrated around $E^{2} \sim M^{2}$, we have, using the linear approximation around $M^{2}$,

$$
\sqrt{E_{n}^{2}}=M+\frac{1}{2 M}\left(E_{n}^{2}-M^{2}\right)+\mathcal{O}\left(E_{n}^{2}-M^{2}\right)^{2} .
$$


This gives the approximation for $H^{1 / 2}$ in the form

$$
\hat{H}^{1 / 2} \approx \frac{M}{2}+\frac{1}{2 M} H
$$

Thus, due to the nature of the spectrum, the equation

$$
i \partial_{\tau} \psi=\hat{H}^{1 / 2} \psi
$$

is well approximated by

$$
i \partial_{\tau} \psi=\left(\frac{M}{2}+\frac{1}{2 M} \hat{H}\right) \psi .
$$

With a change of variables $\psi=e^{-i M \tau / 2} v(x, \tau / 2 M)$ the equation to be solved numerically is

$$
i \partial_{\mathbf{t}} v=\hat{H} v
$$

where $\mathbf{t}=\tau / 2 M$. This reflects the combination of two facts : first that classically $H^{1 / 2}$ and $H$ have the same orbits with rescaled time, and second that since the discrete spectrum only runs over a small energy range, the solution to (51) is well approximated by the solution to (53).

\section{B. Numerical scheme and numerical stability}

We now outline the numerical scheme used for the solutions of the equations. The equation to be solved is (53). These equation was solved numerically using a pseudo-spectral method based on that of [12] for the Korteweg-de Vries equation. This method involves finite Fourier transforms in the $x$-direction, with the equation propagated forward in the $t$-direction by fourth-order Runge-Kutta integration. The finite Fourier transforms are evaluated using fast Fourier transforms (FFT).

The partial differential equation (53) is numerically solved in the interval $-L / 2 \leq x \leq$ $L / 2$. Let us denote the Fourier coefficients of $v$ by $\hat{v}_{n}$ and let $\xi_{n}=2 n \pi / L$. Then the Fourier integral operators in (53) can be evaluated as

$$
\begin{gathered}
\frac{1}{N} \sum_{n=-m}^{n=m} \cosh \xi_{n} \hat{v}_{n} e^{-i \xi_{n} x}, \\
\frac{1}{N} \sum_{n=-m}^{n=m}\left|\sinh \xi_{n}\right| \hat{v}_{n} e^{-i \xi_{n} x}
\end{gathered}
$$

respectively, where $2 m+1$ terms are taken in the finite Fourier series.

Once the Fourier integrals have been evaluated, the partial differential equation (53) is then integrated forward in time in Fourier space. This integration is carried out in Fourier

space due to its superior stability. Let us denote the right hand side of (53) by $G v$. Then if $F$ denotes the finite Fourier transform, we have

$$
\frac{d}{d t} F(v)=-i F(G v)
$$

The ordinary differential equation (56) is integrated using fourth-order Runge-Kutta integration to find $F(v)$. The solution $v$ is then determined by inversion using the FFT. The 
pseudo-spectral method applied to the partial differential equation (53) has an inherent numerical stability problem which will now be discussed.

The interface at $x=2 M$ is a boundary layer in which the solution for $v$ rapidly oscillates. This rapid oscillation results in the formation of high frequency modes in the finite Fourier series. Then, due to the equivalence between small $x$ in physical space and large $\xi_{n}$ in Fourier space, these high frequency modes generate instabilities for $x \approx 0$ which then propagate into the rest of the numerical domain. These high frequency modes are a major problem for (53) as the hyperbolic functions in the Fourier integrals magnify their influence. To reduce these numerical instabilities, the numerical method as outlined above was changed in two ways. The first was that the governing equation (53) was changed to

$$
\begin{gathered}
i \partial_{t} v=2\left\{\left(x^{2}-M x\right) v\right. \\
\left.-x^{3 / 2}\left[(x-2 M)^{2}+\epsilon^{2}\right]^{1 / 4} \frac{1}{2 \pi} \int_{-\infty}^{\infty} \cosh k e^{i k x} \hat{v}(k, t) d k\right\}, \\
x \geq 2 M \\
i \partial_{t} v=2\left\{\left(x^{2}-M x\right) v\right. \\
\left.-x^{3 / 2}\left[(x-2 M)^{2}+\epsilon^{2}\right]^{1 / 4} \frac{1}{2 \pi} \int_{-\infty}^{\infty}|\sinh k| e^{i k x} \hat{v}(k, t) d k\right\}, \\
x \leq 2 M .
\end{gathered}
$$

The modification of the coefficient of the Fourier integral terms in the equations was done to help resolve the boundary (critical) layer. The system (57) and (58) was solved as previously explained in (56). In the numerical solutions, $\epsilon$ was taken to be 0.1 . The second modification was that a low pass filter was applied when evaluating the integrals in (57) and (58). This low pass filter is equivalent to setting $\cosh \xi_{n}$ and $\sinh \xi_{n}$ to 0 for large $\left|\xi_{n}\right|$. It is interesting to note that the concentration of the spectrum at $E \sim M$ allowed us to control the instability by using the very accurate linear approximation given in (53). In other problems it may turn out that the spectrum is not concentrated in a small region, in which case one must find the numerical square root of the matrix $F G$. This process must be carried out in a manner consistent with the filtering, and this procedure is currently being studied. The choice of the number of modes used to obtain an accurate numerical solution was dictated by comparison of the long time numerical solution with the WKB solution as an initial condition with the same W.K.B solution.

\section{Numerical solutions}

We begin by considering the simpler problem of solving Eq. (41) with a Gaussian as an initial condition. In Fig. 3 we present the result for the integration of (41) with the Gaussian initial condition

$$
u(x, 0)=e^{-\left(x-x_{0}\right)^{2} / \lambda},
$$

for the parameter values $\lambda=1.0$ and $x_{0}=7.0$, which was reflected as an odd function about $x=0$ to satisfy the boundary conditions at $x=0$. This figure clearly shows the decomposition of the initial condition into smooth normal modes which are localized away from $x=0$. Notice that the modes are smooth and resemble the hydrogen atom eigenfunctions, as can be seen from equations (44) and (46) and the representation in terms of Laguerre functions (8). 


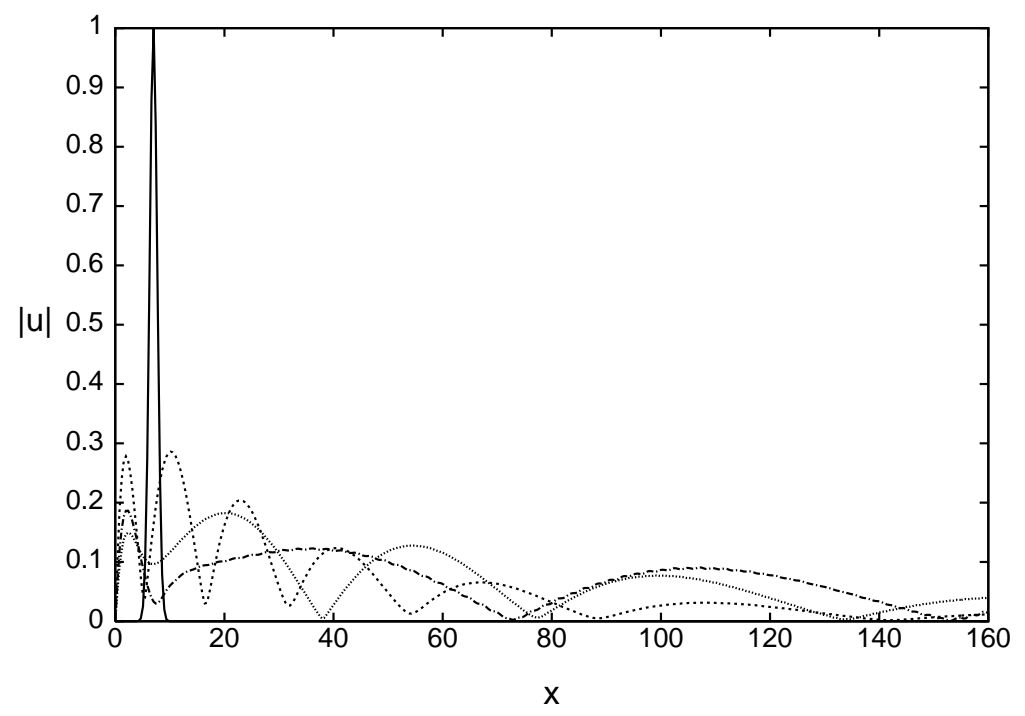

FIG. 3: Numerical solution of model equation (27) for initial condition (59) with $x_{0}=7$ and $\lambda=1.0$. Here $\beta=0.5 . t=0:-; t=20:---; t=50: \cdots ; t=100:----$.

To study the problem posed by Eqn. (53) we begin by assessing the validity of the WKB analysis. To this end we use the WKB eigenfunction of Equations (38) and (39) for $n=2$. This eigenfunction is shown in Fig. 田. In Fig. 5 we show the evolution under the Schrödinger equations (57) and (58) of this WKB solution as an initial condition. It can be seen that a small amount of radiation leaks to infinity and that the WKB solution is distorted by high frequency waves. The high frequency oscillations are due to the non-smooth WKB initial condition and these high wavenumbers then spread under the influence of the non-local nature of the governing equations (57) and (58). Ignoring these high frequency waves, the full numerical solution compares well with the WKB approximation. This good comparison gives confidence in using WKB solutions to explore general initial value problems.

Taking into account the above considerations, let us consider the initial value problem for (57) and (58) with a Gaussian initial condition. We have two cases: $x_{0}>2 M$ and $x_{0}<2 M$. Let us consider first the case $x_{0}>2 M$. In Fig. 5 we show the evolution of the initial condition at $t=5$, at which time the solution has reached the steady state. For $M=5$ this figure shows how the initial value is decomposed into the eigenfunction and a small amount of probability current that is propagated towards infinity. This result can be accounted for by the following WKB analysis.

The discrete eigenfunctions have their oscillatory part concentrated around $2 M$. Thus the Gaussian projected onto these modes decomposes into the oscillations shown in Figs. 6 and 7. The projection onto the continuous spectrum produces the small current which spreads to infinity with decaying amplitude. The high frequency of the radiated waves stems from the short wave behavior displayed in the WKB solutions.

The situation for the opposite case $x_{0}<2 M$ is shown in Fig. 8 The chosen mass parameter was $M=10$ and the initial value was taken to be $x_{0}=4$. Here we have the opposite situation occurring, namely that the evolution is confined to the left of $x=2 M$, while for $x_{0}>2 M$ we found that the solution was concentrated to the right of $x=2 M$. This observation may be explained in terms of the WKB amplitude given in (36) and (37). From those equations it follows that the amplitude $A_{2}$ is small at $x=2 M$ and this, in turn, blocks the passage 


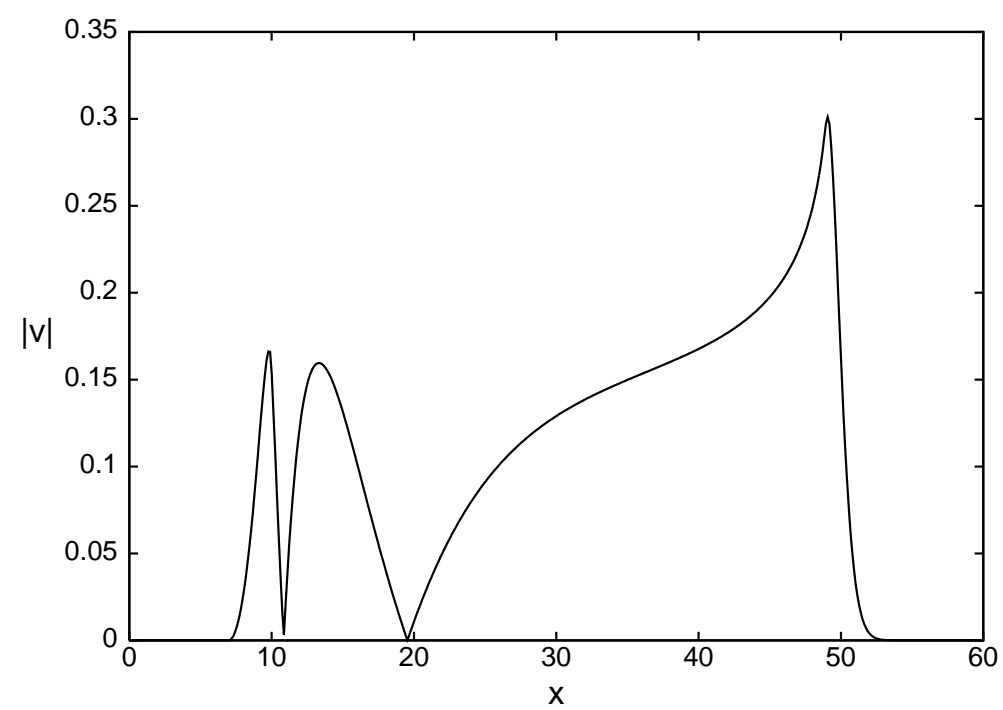

FIG. 4: WKB solution given by (38) and (39) with $M=10$ and $n=3$.

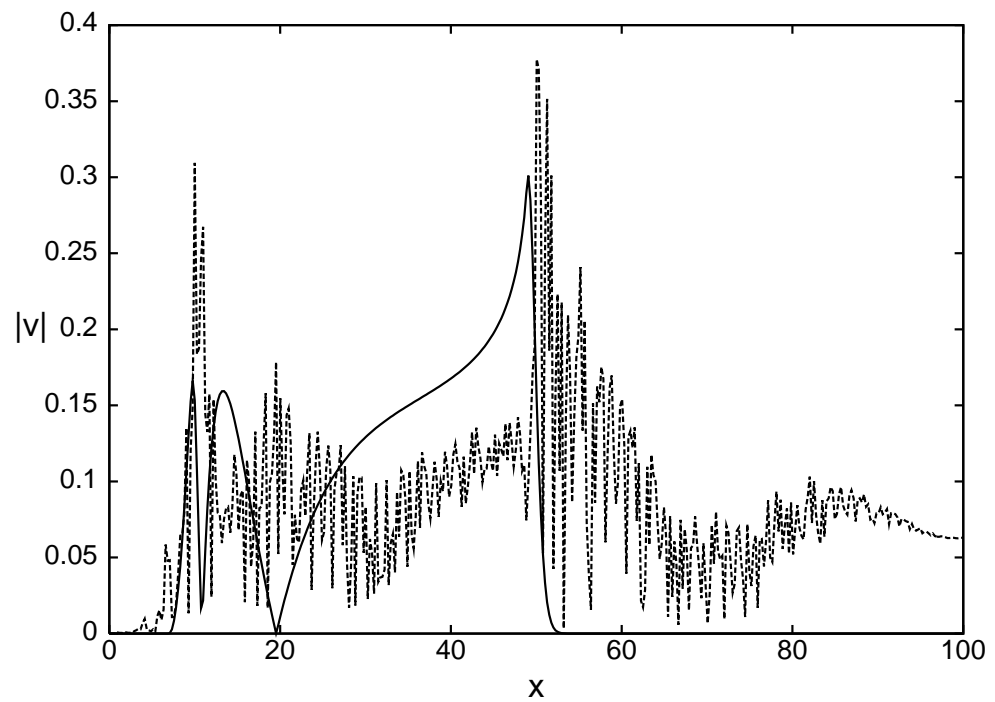

FIG. 5: Evolution of the WKB solution as an initial condition. Numerical solution of (57) and (58) at $t=5$ : --- ; WKB initial condition: - .

of the wave function from one region to the other. The above result can also be understood by noting that at $x=2 M$ the equations lose the highest order derivative responsible for propagation and thus the group velocity goes to zero at $x=2 M$ and the probability current also goes to zero (similar to critical layer absorption at the barrier).

These numerical results confirm our analysis and show that when the radius of the initial shell is larger than its "Schwarzschild radius", the wave function is concentrated outside $x=2 M$. While when the radius of the initial shell is inside the "Schwarzschild radius", the wave function leaks out very slowly. Note that the region $x \approx 0$ is always a region of small probability. Finally, note that these solutions show the evolution of the probability density of the radius of the shell measured as an intrinsic variable. As they stand, they provide no 


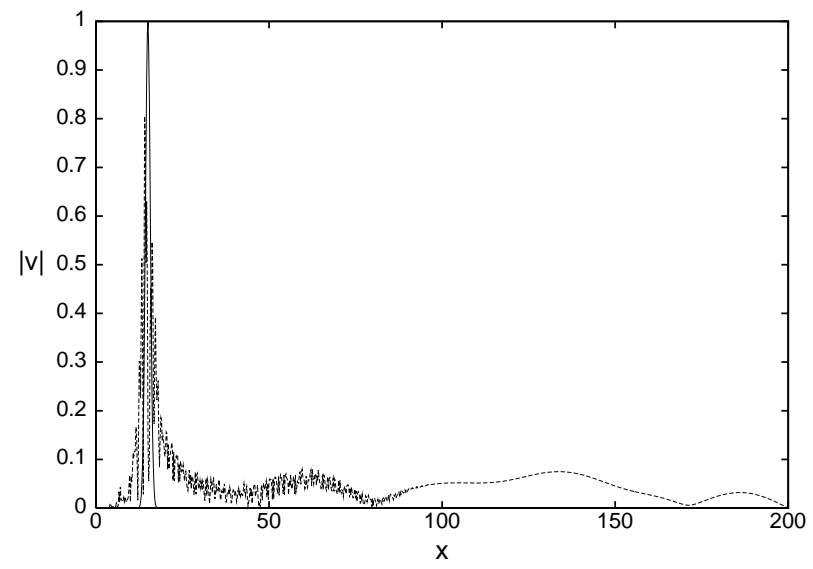

FIG. 6: Numerical solution of (57) and (58) for the initial condition (59) with $\lambda=1.0$ and $x_{0}=15.0$ for $M=5$. Initial condition is outside event horizon at $x=10$. Initial condition: — ; solution at $t=5:---$. Solution in $0<x<200$.

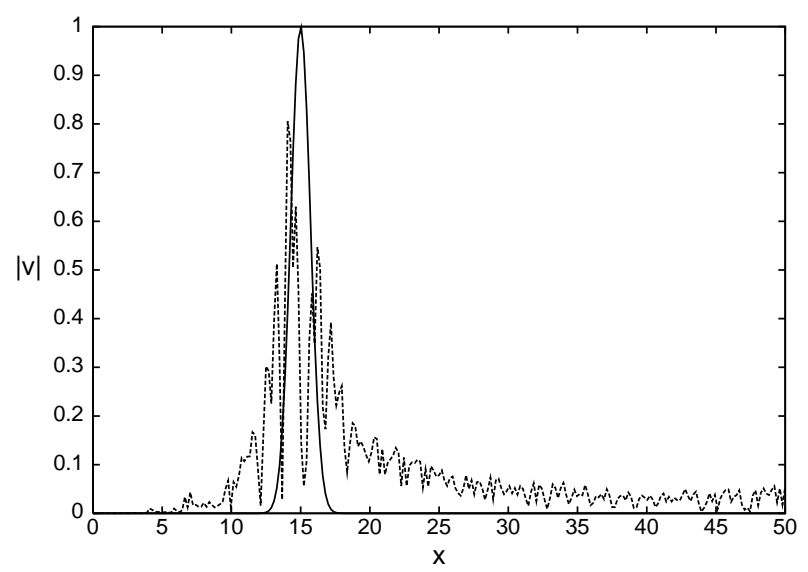

FIG. 7: Numerical solution of (57) and (58) for the initial condition (59) with $\lambda=1.0$ and $x_{0}=15.0$ for $M=5$. Initial condition is outside event horizon at $x=10$. Initial condition: - ; solution at $t=5$ : --- . Solution in $0<x<50$ showing decay behind event horizon at $x=10$.

information about the space-time geometry generated by the collapsing shell. This geometry must be calculated and then quantized making use of the operators generated by the motion of the shell. This is the subject of ongoing work.

\section{CONCLUSIONS}

We have shown how the quantum evolution of the relativistic collapse of a shell of matter can be described by making use of the dimensionally reduced true relativistic Hamiltonian describing this process. In our analysis we have also given a procedure for quantizing a multi-valued Hamiltonian, and we have chosen an appropriate lapse function to produce a tractable Schrödinger equation. This equation has several novel features which were analyzed in the process of constructing the WKB solutions. These solutions have an infinite set of 


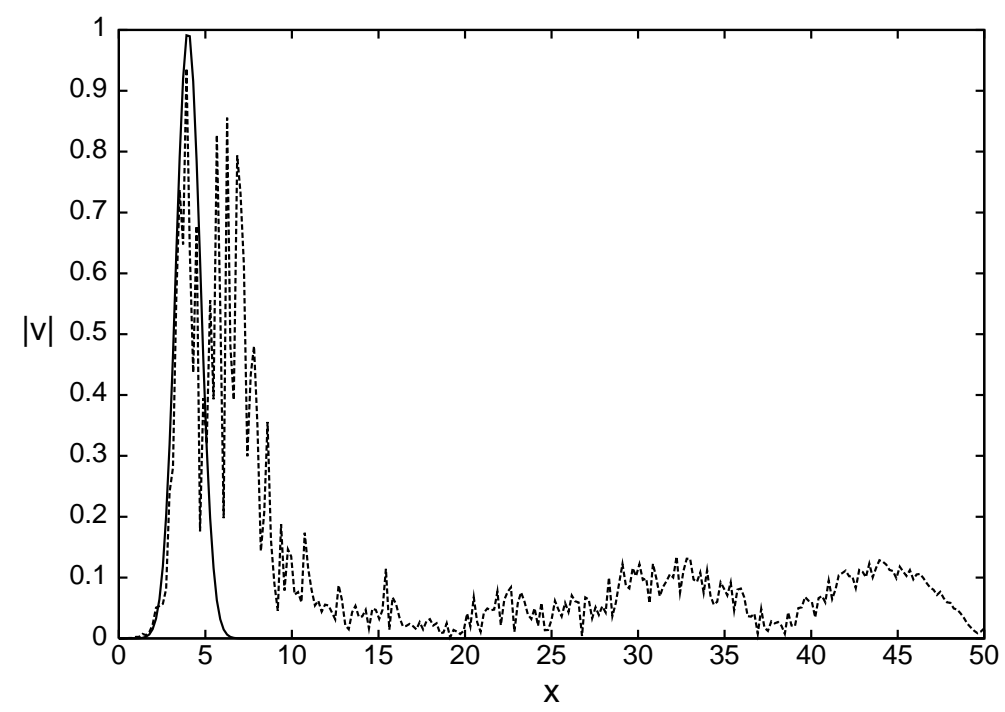

FIG. 8: Numerical solution of (57) and (58) for the initial condition (59) with $\lambda=1.0$ and $x_{0}=4.0$ for $M=10$. Initial condition is inside event horizon at $x=20$. Initial condition: - ; solution at $t=10:---$.

bound states as well as a set of continuum eigenstates. The discrete eigenstates were shown to concentrate away from $R=0$. Thus, in analogy to 15, this suggests that the shell does not enter on average the singularity. The following possibility is also suggested by the present analysis: an incoming wave packet which classically collapses into the singularity $R=0$ now, due to the quantum fluctuations, is bounced back but into a new expanding space-time because of the small quantum fluctuations at $R \sim 0$. This is a consequence of the Uncertainty Principle which in [15] keeps open the throat of the wormhole and in this work bounces the wave function into a new expanding space-time.

The numerical solutions show that the quantum dynamics of an initial condition, representing a Gaussian distribution of shell radii, evolves into a sum over the discrete eigenstates. The critical layer nature of the process is contrasted with the behavior of other ad hoc models appearing in the literature which turns out to be closer to that of the hydrogen atom.

The quantum mechanics developed in this work does not directly answer questions about the quantum geometry of the space. It does, however, set the framework for analyzing the local geometry of the space-time around the shell in terms of the operators defined in this work. In addition, the viewpoint we have presented provides a completely consistent quantization from first principles and a choice of time, related to the proper time on the shell, which explains in WKB terms the behavior of the dynamics observed in previous ad hoc models. It must be stressed that the combination of the WKB approximation and numerics that we used provides a complete description of a consistent quantum dynamics for the radius of collapsing shells of matter in a wide parameter regime.

There are several possible directions for future work. An important study would be to investigate the external geometry of the system as mentioned above and the embedding of the shell in this spacetime. This is not an easy job. In [18], which discusses the collapse of null shells, it was necessary, in order to obtain a tractable problem, to define new canonical variables, and the results for the exterior geometry and embedding were not those that would seem obvious at first glance. Finding the exterior geometry would allow us to understand 
whether the quantum mechanical expansion of the shells we found corresponds to a quantum mechanical tunneling through a horizon or expands into a different spacetime. Another direction would be to study superpositions of states with different values of $M$. This would lead to a "fuzzy" horizon. Note that in our approximation (53), these superpositions will have a very different evolution from the $M$-eigenstate we have used.

Finally, it is important to remark that the results here obtained are within the minisuperspace formalism, where one first reduces the classical system and then quantizes. Recent results in isotropic (loop) quantum cosmology indicate that the inverse strategy, namely to first quantize end then reduce, might yield qualitative different physical predictions at the Planck scale [19].

*

\section{APPENDIX A}

We here consider the transition layer behavior at $x=R^{*}(E)$. To do this, expand Eq. (30) about $R^{*}(E)$ to obtain

$$
f(x, E)=\frac{-E^{2} / 2+x^{2}-M x}{x^{3 / 2}(x-2 M)^{1 / 2}} \approx 1+\frac{\theta^{\prime 2}}{2},
$$

or

$$
1+f^{\prime}\left(R^{*}, E\right)\left[x-R^{*}(E)\right]=1+\frac{\theta^{\prime 2}}{2} .
$$

Now $\theta^{\prime 2}$ is replaced by the second derivative of the boundary layer function, $w$, and we obtain

$$
w^{\prime \prime}+f^{\prime}\left[R^{*}(E), E\right)\left[x-R^{*}(E)\right] w .
$$

Since $f^{\prime}<0$, we have the Airy equation for $w$ [11]. It has solutions of the form

$$
\begin{gathered}
w=\operatorname{Ai}\left(\beta^{1 / 3}\left[x-R^{*}(E)\right]\right), \\
\beta=2\left|\frac{-\left(M^{2}+E^{2}\right) R^{*}+\frac{3}{2} M E^{2}}{R^{* 2}-2 M R^{*}}\right| .
\end{gathered}
$$

The Airy function matches the oscillatory solution as $x-R^{*}(E) \rightarrow-\infty$, and decays exponentially as $x-R^{*}(E) \rightarrow \infty$. In the matching of the phases of the Airy function and $\sin \varphi_{2}(x)$, we obtain the WKB energy quantization rule in the usual way [11].

To consider the matching at the point $\tilde{R}(E)$, we follow the same procedure. Expanding around $\tilde{R}(E)$, we obtain

$$
f^{\prime}[\tilde{R}(E)][x-\tilde{R}(E)] u=\frac{1}{2 \pi} \int_{-\infty}^{\infty}|\sinh k| e^{i k x} \hat{u} d k
$$

Changing variables to $x^{\prime} \equiv x-\tilde{R}(E)$, we have

$$
f^{\prime}[\tilde{R}(E)] x^{\prime} u=\frac{1}{2 \pi} \int_{-\infty}^{\infty}|\sinh k| e^{i k x^{\prime}} \hat{u} d k .
$$

This equation can be solved by means of Fourier transforms to give

$$
i \frac{d}{d k} \hat{u}=|\sinh k| \hat{u}
$$


which yields

$$
\hat{u}=A e^{-i \int_{0}^{k}|\sinh \xi| d \xi},
$$

or

$$
\hat{u}= \begin{cases}A e^{-i(\cosh k-1)}, \quad k \geq 0, \\ A e^{-i(\cosh k+1)}, \quad k<0 .\end{cases}
$$

Note that this function is not analytic at $k=0$. Thus, the only path of integration for the inversion is the real axis. We obtain

$$
u\left(x^{\prime}\right)=\frac{A}{2 \pi} \int_{0}^{\infty} e^{i k x^{\prime}} e^{-i(\cosh k-1)} d k+\frac{A}{2 \pi} \int_{-\infty}^{0} e^{i k x^{\prime}} e^{i(\cosh k-1)} d k .
$$

For $x>0$ the points of stationary phase satisfy

$$
|\sinh k|=x^{\prime},
$$

as they should to match the behavior for $x>0$. On the other hand, for $x<0$ there are no points of stationary phase. The integral, after an integration by parts, shows that $u$ decays as

$$
u(x) \sim \frac{\text { constant }}{x^{\prime}} \quad \text { as } x^{\prime} \rightarrow-\infty .
$$

This is due to the nonanaliticity of $|\sinh k|$.

This behavior shows us the reason for the sharp decay of amplitude observed in the numerics. Note that although this analysis is only local around $x=\tilde{R}(E)$, it is sufficient to complete the WKB solution and to explain the numerics.

\section{ACKNOWLEDGMENTS}

We would like to thank C. Beetle, J. Guven, and B. Whiting for discussions, and specially Karel Kuchař for many fruitful discussions during the various stages of this work. This work was supported in part by UNAM-DGAPA Project No. IN 106097 and by CONACyT Project No. G 25427-E and J32754-E. Our special thanks to Ana Guzman for her help in computations.

[1] L.D. Landau and E.M. Lifshitz, Quantum Mechanics, Addison-Wesley, 1958.

[2] P. Hájíček, Comm. Math. Phys. 150, 545 (1992).

[3] K. Kuchař and P. Hájíček, Canonical dynamics of gravitating shells, Preprint.

[4] P. Hájíček, B. Kay and K. Kuchař, Phys. Rev. D, 46, 5439 (1997).

[5] J.L. Friedman, J. Louko, S.N. Winters-Hilt, Phys. Rev. D, 56, 7674 (1997).

[6] W. Israel, Nuovo Cimento 44B, 463 (1967).

[7] P. Hájíček and Bičák, Phys. Rev. D, 56, 4706 (1997).

[8] V.A. Berezin, Int. J. Mod. Phys., D5, 679 (1996).

[9] V.A. Gladush, J. Math. Phys., 42, 2590 (2001).

[10] G.B. Whitham Linear and Nonlinear waves, John Wiley, 1976.

[11] A.A. Minzoni and T. Miloh, Wave Motion 20, 131 (1994). 
[12] B. Fornberg and G.B. Whitham, Phil. Trans. Roy. Soc. London A, 289, 373 (1978).

[13] E. Farhi, A.H. Guth and J. Guven, Nucl. Phys. B 339, 417 (1990).

[14] J. Lighthill, Waves in Fluids, Cambridge University Press, Cambridge, 1978.

[15] M. Visser, Phys. Rev. D 43, 402 (1991).

[16] A. Ansoldi, A. Aurilia, R. Balbinot, and E. Spallucci, Phys. Essays 9, 556 (1996).

[17] A. Ansoldi, A. Aurilia, R. Balbinot, and E. Spallucci, Class. Quantum Grav. 14, 1 (1997).

[18] J. Louko, B. Whiting, and J. Friedman, Phys. Rev. D 57, 2279 (1998); B. Whiting private communication.

[19] M. Bojowald, Phys. Rev. Lett. 86, 5227 (2001).

[20] recall that a $c$-number is a number independent of the phase space point. A phase space dependent number (constant of the motion) is called a $q$-number. 